\title{
Contact lenses: clinical evaluation, associated challenges and perspectives
}

\begin{abstract}
Objectives: The continuous interest surrounding the utilisation of contact lens for various purposes such as vision correction, cosmetics, and ocular drug delivery has led to more innovations to maximise its potential into more novel applications. Contact lens serving as a polymer matrix vehicle may be an effective system in releasing drugs at a desired rate and duration, proving supreme over conventional methods. Although studies are continuously conducted to gather evidence of its potential and effectiveness, there are various hurdles preventing availability to the public.
\end{abstract}

Methods: This review surveys the current and potential applications of contact lenses with emphasis on in vivo studies, clinical trials and current regulations.

Key findings: The number of contact lenses' wearers has gradually increased overtime. With the continuous research advancement, there is a high hope for contact lenses to be used as an ocular drug delivery system and could potentially provide a great platform for managing conditions such as glaucoma, allergy and photosensitivity. Moreover, the use of smart contact lenses in managing non-ocular conditions like diabetes demonstrates its endless possibilities.

Conclusion: Side effects associated with frequent using of contact lenses and current regulations by governing bodies are amongst the challenges for the emerging new applications.

Keywords: contact lenses, applications, hazards, regulations, clinical evaluation, in vivo studies, glaucoma, allergy, photosensitivity, staphylococci, pseudomonas, acanthamoeba

\author{
Volume 5 Issue 3 - 2017
}

\author{
Reynalyn Mosuela,' Asim Thapa,' Raid G \\ Alany, ${ }^{1,2}$ Amr ElShaer ${ }^{1}$ \\ 'Drug Discovery, Delivery and Patient Care (DDDPC), Kingston \\ University London, UK \\ ${ }^{2}$ School of Pharmacy, the University of Auckland, Auckland, New \\ Zealand
}

Correspondence: Amr ElShaer, Drug Discovery, Delivery and Patient Care (DDDPC), School of Life Sciences, Pharmacy and Chemistry Kingston University London, UK, Tel +44 (0)20 84I7 7416,Email a.elshaer@kingston.ac.uk

Received: April 06, 2017| Published: April 21, 2017
Abbreviations: PMMA, poly methyl methacrylate; HEMA, hydroxyethyl methacrylate; FDA, food and drug administration; BCLA, british contact lens association; ACHIEVE, adolescent and child health initiative to encourage vision empowerment; UDVA, uncorrected distance visual activity; IOL, intraocular lens; IOP, intraocular pressure; MEMS, micro electro mechanical system; AOA, american optometric association; ANSI, american national standards institute; MHRA, medicines and healthcare products regulatory agency; SFDA, state food and drug administration

\section{Introduction}

The evolution of contact lenses goes back to the $15^{\text {th }}$ century when Leonardo da Vinci first proposed theories of how a water-filled glass could neutralize light bending property or refractive power of the cornea to improve image clarity. ${ }^{1}$ The first contact lens was prepared by Thomas Young using wax. Over theyears contact lenses polymers were developed starting with the use of oxygen impermeable poly methyl methacrylate (PMMA) as material for scleral lens ${ }^{2}$ to the development of the first soft hydrogel; poly hydroxyethyl methacrylate (HEMA) which was the first contact lens polymer to be approved by the Food and Drug Administration (FDA). Despite achieving success, practitioners discovered that the extended wear of contact lenses develops corneal oedema due to disproved oxygen transmission. Hence, soft contact lenses were re-classified as medical devices under the Medical Device Amendments in 1979. ${ }^{3-6}$ It was later discovered that the high water absorbency of HEMA-containing hydro gels made it not only difficult to handle but also gave rise to, low oxygen permeation and adherence of bacteria resulting into eye infections. This led to the discovery of hydrophobic materials such as cellulose acetate butyrate ${ }^{7}$ and siloxane-containing polymers. ${ }^{8}$ The present developments on contact lenses promise its use as a potential ocular drug delivery system for clinical therapeutics. However, regulatory issues are still to be met and satisfied before medicated contact lenses can reach the market. ${ }^{9}$

\section{Purposes and applications of contact lenses}

Contact lens was first invented to protect the eye, improve the clarity of the images and correct vision. Overtime, they been more proven to be more effective than the conventional eye glasses; hence increasing preference in wearers. ${ }^{10,11}$ Nowadays, contact lenses are categorized according to their use in optical applications. These are corrective, non-corrective, medicated, and other potential applications.

\section{Corrective}

Contact lens, in terms of correcting vision, functions the same way as conventional eyeglasses. ${ }^{12}$ There are, however, different types of contact lenses to correct different vision problems including myopia (near-sightedness), hyperopia (far-sightedness), and astigmatism (both near and far-sightedness. ${ }^{13}$

\section{Myopia}

Myopia is one of the prevailing causes of reduced vision throughout the world. In simple terms, myopia or near-sightedness is the blurred vision to far away objects. According to the British Contact Lens Association (BCLA), myopia develops during the early ages of a growing adult, usually from 6 to 8years of age although the onset of progression varies. ${ }^{14}$ Theoretically, myopia is a result of changes concerning the length of eyeball. ${ }^{15}$ When the eyeball is affected by 
the change in shape of the cornea for prolonged periods, this causes the light rays to focus at a point in front of the retina rather than on its surface directly. Diagnosis and treatment of myopia involve the expertise of an eye specialist to provide best method of treatment. Eyeglasses are the simplest way to correct this vision problem. However, contact lens gives more benefits, mainly comfort and clearer vision along with a more cosmetic appeal. ${ }^{16}$ The main types of recommended lenses are gas permeable, soft, bifocal, multi focal and orthokeratology. ${ }^{14}$ However, the use of these methods to correct vision issues was not enough for long term management resulting in a probable reoccurrence of myopia. Hence, different studies have been conducted to assess the efficacy of contact lenses and other vision corrective methods to control the progression rate of myopia, especially to young age groups. A study was conducted to determine the effect of soft multifocal lenses on the progression of myopia in children from 8 to 11 years of age $(n=40)$ with a -1.00 to $-6.00 \mathrm{D}$ spherical component and less than $1.00 \mathrm{D}$ astigmatism over a 2-year treatment. The study revealed that the use of contact lens reduced the progression rate of myopia by $50 \% .{ }^{17}$ Even though studies showed that contact lenses aid in the slowing of myopic progression, it is essential to give patients the best fit of contact lenses; the type and the suitability of the lens to the wearer. Different lenses used to slow down the progression of myopia were discussed in a review. ${ }^{18}$ Gas permeable contact lenses (GP contact lenses) improve the retinal image and flattening of the cornea. The utilisation of GP contact lenses in slowing down the progression rate of myopia was tested in a 2year randomized controlled trial to which its purpose was to appraise the efficacy of rigid GP contact lenses to reduce the rate myopic progression to Singaporean children $(n=428)$ of ages 6-12years. ${ }^{19}$ Based from this trial, there was a slight reduction of progression rate but it was not significantly reduced even to children who used the lenses regularly. However, even if the lenses were not the recommended option for controlling myopic progression, its invaluable vision correction purpose was significantly recognized. A popular type of contact lens, soft contact lens was also tested to see whether it can be used to slow myopic progression in an Adolescent and Child Health Initiative to Encourage Vision Empowerment (ACHIEVE) study. ${ }^{20}$ This randomized study was conducted over a span of 3years amongst ethnically diverse children $(n=484)$ with a mean age of 10.4 \pm 1.1 years. Based from the 3year trial, use of contact lenses did not show any significant increase clinically in myopic progression. ${ }^{\mathrm{s}}$ Multifocal soft contact lens is another type of lenses that do not just correct vision, but provide a promising reduction on myopic progression. ${ }^{18}$ An update was carried out by Guthrie in $2011^{21}$ and evaluated the effectiveness of bifocal contact lenses to decrease the pace of myopia progression. ${ }^{21}$ Guthrie's update focused on children $(\mathrm{n}=40)$ aged 11-14years for a 10month treatment (Phase 1) with single vision distance lenses, and then bifocal lenses cross over assignment for another 10months (Phase 2). The study revealed that there was less myopic progression after Phase 2 than in Phase 1. It was also revealed that myopic progression was reduced significantly when dual focus lenses were used to children $(n=34)$ who successfully completed the study with a progression reduction of $0.20 \mathrm{D}(54 \%)$. Moreover, It was reported that dual focus lenses offered better acuity and contrast sensitivity. ${ }^{21}$ There are still different methods for controlling myopic progression, ${ }^{18}$ but the focus of this review remains on contact lenses.

\section{Hyperopia}

Hyperopia or far-sightedness is another type of refractive error that is opposite of myopia. It occurs when distant objects are clearly seen, but near objects appear to be blurred. Holding theoretical similarity to myopia, hyperopia is experienced when there are irregularities on the length or shape of the eyeball. This prevents the light from focusing on the retina, hence the light is focused behind the retina. ${ }^{22,23}$ Hyperopia is usually hereditary but in rare cases, it is caused by other conditions including diabetes, small eye syndrome, and cancers surrounding the eye. The diagnosis and treatment for hyperopia are similar to those of myopia. There are limited studies on the efficacy of contact lenses as mode of treatment for reducing the progression rates of hyperopia. However, there was a study conducted to test the contact lens to correct hyperopia without the need of surgery. This experimental study consisted of patients $(n=10)$ with hyperopia aiming to treat this condition without the need of ortho-K technology or corneal reshaping. In this study, patients wore designed contact lenses only at night and not during the day. The mechanism as to how this new contact lens worked was by application of pressure to the tear film which would in turn change the shape of the cornea. ${ }^{24}$ Its potential use in hyperopia correction was assessed and confirmed in this study. Another novel treatment using a secondary implantation of a collamer lens was shown in a study for the correction of anisometropic hyperopia in a three year old baby. ${ }^{25}$ Although not a typical contact lens and requires surgery, this new variation displayed some promising results in infants with such condition. Not only did the child show a significant improvement in terms of uncorrected distance visual activity (UDVA) postoperatively (20/400 to 20/50), but there were no vault complications and intolerances observed on a 22 month follow-up. Hence the potential use in hyperopia correction can be confirmed with a collection of above studies. ${ }^{25}$ Another retrospective breakthrough is the method of intraocular lens (IOL) implantation to correct hyperopia. A three-year follow-up study was conducted by Siganos et al in 1994 that showed promising results of the procedure in 17 subjects of ages ranging from 35 to 55 years diagnosed with hyperopia of a spherical equivalent of $+9.61 \pm 0.46 \mathrm{D}^{25}$ The study revealed that there were complications of IOL implantation occurred during the threeyears span post-treatment. However, the benefits of this procedure outweighed the risks in terms of unaided visual acuity improvement, hyperopic correction, reduced percentage of endothelial cell loss and overall stability and safety. From the results and findings of this study, it was significant to take into account other ophthalmic conditions before delivering the procedure and the provision of more clinical studies that would utilise other types of lenses including multifocal lenses to achieve maximum hyperopic correction and could override other refractive procedures such as surgery. ${ }^{26}$

\section{Astigmatism}

Astigmatism is another type of refractive error where the eye does not focus light evenly onto the retina. It is caused by the change in corneal shape where there is varied level of roundness in the corneal areas which results to difference in light bending. ${ }^{26,27}$ The diagnosis of astigmatism is carried out following a comprehensive dilated eye exam. Consequently, the treatment for astigmatism is the same as that of myopia and hyperopia where GP contact lens is the most suitable type of lens for correction. This lens is capable of moulding the tear film in front of the eye into a spherical shape credit to the material's rigidity. ${ }^{29}$ On the other hand, soft contact lenses are not suitable for astigmatism because it loosely covers and sits over the cornea, hence failing to correct vision. However, a specially designed soft lens called 'toric' lens does help with vision correction. Toric lenses are designed with one axis that is able to correct more refractive error than the other. ${ }^{28,29}$ They provide better visual acuity as 
compared to conventional spectacles. Nowadays, there are a variety of developments on toric soft lenses which aim to further improve the stability and performance in correcting astigmatism. ${ }^{30}$ One of them is the introduction of silicone hydro gels as toric lenses. Silicone hydro gels are good toric lenses because the silicone component of the lens enhances oxygen permeability and is showed to decrease the prevalence of complications during treatment. ${ }^{30}$ However, in a study conducted in $2011,50 \%$ of spectacle wearers in the UK $(n=11000)$ showed astigmatism development in one or both eyes and one-third of the total population of soft contact lens wearers require astigmatism correction via toric soft contact lenses in either one or both eyes. ${ }^{31}$ In 2000, the Food and Drug Administration initiated the regrouping of hydrogel contact lenses in terms of their properties namely water content and chemical properties. ${ }^{7}$ In particular, spherical hydrogel contact lenses were used as corrective lenses for the treatment of myopia and hyperopia which are both discussed above. ${ }^{32}$ These groups are 1,2, 3 and 4. Groups 1 and 2 have both non-ionic chemical properties, but differ in water content where Group 1 exhibits $<50 \%$ water content and Group 2 with $>50 \%$ water content. Both Groups 3 and 4 show ionic chemical properties, and again, differ in water content. Group 3 has $<50 \%$ water content whilst Group 4 shows $>50 \%$ water content. Some examples of contact lenses approved by the FDA are listed in Table 1.

With regards to the market share of the different types of contact lenses i.e. Daily disposable soft contact lenses, soft frequent replacement lenses, traditional soft contact lenses, and hard contact lenses were evaluated in 2013 as shown in Figure 1. The UK CL market equals to $£ 240$ million as reported in 2013; this was a result of sale of over $£ 600$ million. ${ }^{33}$ It is clear that daily disposable contact lenses have gained great popularity lately and this could be attributed to the low infections incidents associated with wearing disposable contact lenses compared to hard contact lenses and soft frequent replacement. This was reflected in a 15 year prescribing behaviour of opticians ${ }^{34}$ with more public acceptability to daily disposable contact lenses. The 3year-long study conducted by Erik et al (1994) revealed that daily wear contact lenses contributed to 0.16 incidents of microbial keratitis in every 10,000 wearers compared to 3.12 for those who use wear conventional contact lenses for extended periods. ${ }^{35}$ Moreover, conventional lenses are associated with accumulation of deposits leading to uncomfortable wearing, impaired visual acuity and higher chances for infections. ${ }^{36}$ The hassle of daily cleaning and disinfection make it unfavourable option to users

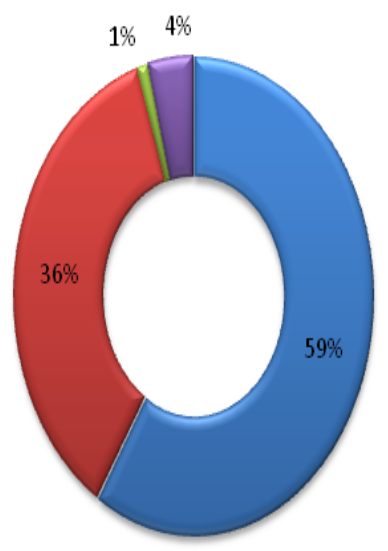

Daily disposable soft

Soft frequent replacement

$\square$ Traditional Soft

Uhard contactlenses

Figure I A brief overview of the share of different type of contact lenses in $2013 .^{33}$
Table I Examples of contact lenses approved by FDA with brand name(s) and manufacturer(s). ${ }^{7,30,32,38-48}$

\begin{tabular}{|c|c|c|}
\hline $\begin{array}{l}\text { Lens } \\
\text { material }\end{array}$ & $\begin{array}{l}\text { Brand } \\
\text { name(s) }\end{array}$ & Manufacturer(s) \\
\hline \multicolumn{3}{|c|}{ Group I (Non-ionic, low water content) } \\
\hline Crofilcon A & CSI EW & Sola/Barnes-Hind Inc, San Diego CA \\
\hline Lotrafilcon A & Night \& Day & $\begin{array}{l}\text { Alcon Laboratories, Inc, United } \\
\text { States (200I) }\end{array}$ \\
\hline Tetrafilcon A & Preference & $\begin{array}{l}\text { Coopervision Ophthalmic Products, } \\
\text { United States (1976) }\end{array}$ \\
\hline Hefilcon B & Optima Toric & Bausch \& Lomb \\
\hline Halyfilcon A & $\begin{array}{l}\text { Acuvue } \\
\text { Advance }\end{array}$ & Johnson \& Johnson Medical \\
\hline
\end{tabular}

Group 2 (Non-ionic, high water content)

$\begin{array}{lll}\text { Alphafilcon A } & \text { Soflen66 } & \text { Bausch \& Lomb } \\ \text { Nelfilcon A } & \text { Focus Dailies } & \text { Ciba vision/ Alcon } \\ \text { Omafilcon A } & \text { Proclear } & \text { Cooper Vision } \\ \text { Hioxifilcon A } & \text { Satureyes } & \text { Metro optics. inc }\end{array}$

\section{Group 3 (lonic, low water content)}

$\begin{array}{lll}\text { Balafilcon A } & \text { Purevision } & \text { Bausch and lomb } \\ \text { Bulifilcon A } & \text { Soft Mate B } & \text { Wesley-Jessen Vision Care, Inc. } \\ \text { Ocufilcon A } & \text { Tresoft } & \text { United Contact Lens } \\ \text { Phemfilcon A } & \text { Fresh Look } & \text { Alcon }\end{array}$

\section{Group 4(Ionic, high water content)}

\begin{tabular}{|c|c|c|}
\hline Etafilcon A & Acuvue & Johnson \& Johnson Medical \\
\hline Focofilcon A & Fre-flex & Op tech, Inc \\
\hline Methafilcon & Frequency 55 & $\begin{array}{l}\text { Westcon Contact Lens Co., Inc } \\
(2000)\end{array}$ \\
\hline \multirow[t]{2}{*}{$A, B$} & Soft-Form 55 & Unilens Corp, USA (1989) \\
\hline & & Lombart Ltd (1989) \\
\hline Ocufilcon F & $\begin{array}{l}\text { Hydrogenics } \\
60\end{array}$ & Cooper vision \\
\hline
\end{tabular}

\section{Non-corrective}

Non-corrective lenses are also termed as decorative, 'planocosmetic' or zero-powered contact lenses. Decorative contact lenses are solely for the purpose of changing the appearance of the eye, particularly its colour. Decorative lenses are mostly used in fashion, Halloween cosmetics, and theatre to temporarily alter the appearance i.e. colour of the eyes. These lenses are not manufactured to correct the vision however, they are still recognised, categorised and regulated by the Food and Drug Administration (FDA) ${ }^{36}$ in United States similarly as corrective lenses. This is because decorative lenses may still carry potential risks of infections to the eye if used without care and security. These risks may include potential corneal abrasion, allergic reactions, decreased vision, infection, and probable blindness. These risks are likely to be presented with redness of the eyeball, pain in the eye, or decreased vision. ${ }^{49-51}$ With the increased market for decorative lenses, the Food and Drug Administration has published a guidance document on the regulation of decorative lenses as medical 
devices. ${ }^{51}$ The study conducted by Steinemann et al. ${ }^{52}$ surveyed 159 contact lens wearers, $23 \%$ of whom used plano decorative contact lenses. Twelve patients were admitted to hospitals with acute eye pain and were found to use decorative contact lenses that were not dispensed by eye care professionals. Four patients were infected with staphylococci, Pseudomonas, and acanthamoeba and one patient had to undertake a penetrating keratoplasty. A second study conducted by Singh et al. ${ }^{53}$ demonstrated that wearing coloured cosmetic contact lenses was associated with infectious keratitis. Thirteen young emmetropic individuals caught bacterial and viral infections from decorative contact lenses amongst which 8 youngsters developed corneal ulcers with area greater than $25 \mathrm{~mm}^{2}$ and suffered impaired vision after treatment. It is understood that the risk of developing microbial keratitis raises by 12 -fold because of the inappropriate supervision of using cosmetic contact lenses, while the lack of handling and caring information about the lenses increase the risk by 20 -fold. ${ }^{54}$ This essentially means that even decorative lenses should require a valid prescription from a registered health care professional who holds a registered accreditation for prescribing such devices. In the UK, a valid prescription is not legally required for decorative lenses, but these must be supplied by or under the supervision of a medical practitioner. The British Contact Lens Association (BCLA) explains the current regulations on the use of decorative lenses and its potential risk..$^{55}$

\section{Medicated contact lenses}

In line with the development of contact lens for the purpose of correcting different refractive errors or vision problems, the incorporation of medicines in the lenses has been a novel and interesting field of research up to the present times. Medicated contact lenses, also termed as "smart" contact lenses came into the picture when conventional ophthalmic drug delivery systems such as eye drops and ointments release drugs uncontrollably and $95 \%$ of the drug is lost either into the tear drainage or absorption in the conjunctiva. ${ }^{56,57}$ The incorporation of drugs into contact lenses has faced many challenges before reaching a successful stage. One of obstacles was the rapid drug release and inability to sustain the drug particles into the polymer matrix hence; the contact lenses could only be used over a very short period of time. With further development, strategies to sustain the drugs were introduced extending the drug delivery over a longer period of time. Incorporation of drug molecules in nanoparticles, micro emulsions, and cyclodextrins were used to fulfil this character of the lens. ${ }^{56,58,59}$ However, regulatory agencies have not issued a profound regulation that would categorise medicated contact lenses although they are already working on this. ${ }^{60}$ It is also worth emphasising those contact lenses as a platform for ocular drug delivery is still being continuously studied up to present time. Several studies have already conducted to evaluate the efficacy of silicone hydrogel contact lenses to extend the delivery of ophthalmic drugs such as timolol, dexamethasone, and ciprofloxacin. These experimental studies have shown potential for a drug delivery system, however its stability and safety in vivo must be further studied. ${ }^{61-63}$

A clinical study was carried out by the Department of Ophthalmology in India which included patients $(n=466)$ who were awaiting a surgery to treat their senile cataract at the time. The experimental method of this study involved treatment of the senile cataract using two methods: sub conjunctival injection or drug delivery through contact lenses. Soft contact lenses namely New Sauflon 70 and New Sauflon 85 were soaked in antibiotic solution.
The study had brilliant results. It was shown that the soft contact lenses gave a significantly better penetration even in comparison to the sub conjunctival injection therapy ${ }^{64}$ It is possible that medicationincorporated contact lenses will be regulated and marketed effectively to provide effective treatments in the near future. However, the quest for the ideal contact lens to deliver medications is still a challenge and is associated with the requirements such as controllable zero-order release profiles, shape retaining and consistent acuity, stability on storage and transportation, safe therapeutic drug release profile and good oxygen permeability to enable extended wear. ${ }^{65,66}$

\section{Other potential applications}

Since the development of medicated contact lenses is already pacing forward very quickly, different pharmaceutical companies have introduced other potential applications. One of the first breakthroughs of smart contact lens is its use in the measurement of intraocular pressure (IOP) in the eye. A micro electro mechanical system (MEMS) was developed to measure the changes in IOP of patients with glaucoma; the most common eye disorder clinically presented by an increase in IOP ${ }^{67} \mathrm{~A}$ new contact lens was designed to continuously measure IOP by embedding a pressure sensor in the contact lens polymer matrix. These newly designed contact lenses were tested to volunteers $(n=12)$ who were assessed to have healthy pair of eyes. The procedure consisted of a series of tests such as heart rate, blood pressure, and arterial pressure to monitor the vitals of the subject volunteers before conducting the test. Measurements of IOP were done in both sitting and supine positions. Three conformations of dynamic contour tonometer namely sit-lamp mounted, hand-held, and contact lens were embedded in the sensor. Comparison of the three methods revealed the potential for contact lenses to be an effective system for monitoring IOP over a period of time. ${ }^{67} \mathrm{~A}$ comparative study was conducted by Anton et al to measure the intra ocular pressure using two methods namely Icare Tonometer (Icare ${ }^{\circledR} T A 011$, Finland) and Air puff tonometer (Nidek NT 53OP, Japan) in patients $(n=23)$ with glaucoma $(n=7)$ who were not treated with any topical medication 5 hours before contact lens insertion. Pure Vision 2 HD (Bausch and Lomb, Germany) was used as the contact lens worn by the subjects. It was statistically determined that both methods showed accuracy in measurement of IOP with or without soft contact lens. Air puff tonometer had a mean value of $15.6+2.6 \mathrm{mmHg}$ with lens measurement and $15.3 \pm 2.6 \mathrm{mmHg}$ without lens measurement hence no significant difference was observed. The results for Air puff tonometer showed that there was no correlation with the central corneal thickness. On the other hand, using the Icare tonometer revealed data of $17.5 \pm 4.3 \mathrm{mmHg}$ with lens measurement and $16.4+3.5 \mathrm{mmHg}$ without. As the IOP values using Icare tonometer was higher, it showed a significant correlation with the central corneal thickness which meant that an increase in corneal thickness by $100 \mu \mathrm{m}$ results in subsequent increase in IOP by $3 \mathrm{mmHg} .{ }^{68}$ The first ever "smart" contact lens was designed by biomedical engineers from the University of California, US Tingui Pan and Hailin Cong. The designed "smart" contact lens was made of liquid Poly (dimethylsiloxane) PDMS solution and a chemical to polymerise the mixture upon exposure to UV light. ${ }^{69}$ Addition of silver to the PDMS solution delivered better electrical conductivity for the finished material. This formulation of contact lens made Pan and Cong work with the director of Glaucoma Services at UC Davis Medical Centre to produce a pressure sensor that is bent into the shape of contact lens and can measure the fluid pressure within the eye ${ }^{69,70}$ A pharmaceutical 
company, Sensimed developed a bifunctional wireless version of MEMS incorporated into the smart contact lens. This type of lens includes a MEMS device along with an antenna, a processing circuit and a transmitter for communication. The measuring device named as the SENSIMED Triggerfish ${ }^{\circledR}$, is worn by patients for up to 24 hours including perceived sleeping time. This measuring device monitors natural changes of the eye..$^{71,72} \mathrm{~A}$ clinical study was also conducted in $2011^{73}$ to monitor IOP in glaucoma patients $(n=15)$ using a wireless ocular telemetry sensor (Sensimed AG, Switzerland). Sensimed is a silicone based contact lens embedded with MES and an antenna. 87\% $(n=13)$ of the total sample population completed the IOP monitoring activity where $69 \%$ have recorded the highest signals during sleeping time. Safety, functionality and serious adverse effects of the OTS were assessed. The OTS was found to be safe and no adverse effects with only one subject discontinued due to intolerance. In this clinical study, Sensimed was considered to be of high potential for IOP monitoring for a larger population. ${ }^{73}$ Aside from monitoring the IOP to patients with glaucoma, another research innovation is the Google smart contact lens, which is still being furnished up to this date. Swiss pharmaceutical firm called Novartis and Google Inc. has collaborated to produce this new contact lens. The Google smart contact lens is designed to measure the glucose levels in tear fluid which would help monitor the condition of diabetic patients without the need of taking blood. This was believed to improve the compliance of diabetic patients. This Google smart contact lens uses wireless chip and miniaturised glucose sensor fixed in between two layers of soft contact lenses. Although this was just introduced in early 2014, Google Inc. and Novartis are working together with the Food and Drug Administration (FDA) to bring this product in to market. ${ }^{74}$ Another new development on "smart" contact lenses is the UV-absorbing contact lens. Excess UV exposure can lead to pathological eye conditions such as photo keratitis, photo conjunctivitis, and cataracts. These are caused by the lack of protection of the cornea which is vulnerable to tissue damage when exposed to excess UV light. ${ }^{75-77}$ According to the World Health Organization, an estimation of about 15 million people worldwide become blind annually due to the development of a cataract caused by overexposure to sun. Hence, the American Optometric Association (AOA) recommends the use of eye protective equipment such as sunglasses to block UV and protect the eyes from radiation. However, not all sunglasses are able to protect the eyes fully, thus recommendation of contact lens was made possible. The use of contact lens provides an additional protection by absorbing UV radiation. Moreover, the American National Standards Institute (ANSI) Z80.20 provided standards on the safe use of contact lenses as UV-blocking materials. These standards were then used by the Food and Drug Administration (FDA) in the US to regulate such materials. Two classifications of UV-blocking lenses are: FDA Class I blocker and FDA Class II blocker. Class I blockers comprise of lenses that can block $90 \%$ of UV-A and $99 \%$ of UV-B rays of wavelengths 316-380nm and 280-315nm respectively. An example of Class I UVblocking contact lens approved by FDA is VISTAKON® (senofilcon A). On the other hand, Class II blockers are lenses that block $70 \%$ of UV-A and $95 \%$ of UV-B. ${ }^{75-77}$ To obtain evidence on the UV-blocking properties of contact lenses, an in vivo experiment ${ }^{78}$ was carried out by Giblin et al on UVB- induced ocular tissues of sample rabbits. ${ }^{78}$ Giblin et al administered Xylazine and Ketamin hydrochloride into the subjects before UVB- irradiation. The subjects were then exposed to UVB for half an hour with either UV-blocking Senofilcon A contact lenses or the UV-blocking Lotrafilcon A contact lens. The control of the study was without the use of contact lens. The corneas were stained with fluorescein 15 hours post exposure. The results showed that the subjects in the control group exhibited loss of epithelial cell in the cornea, swelling of the lens epithelial cell, and breaking of DNA single strands. On the other hand, the eyes with Senofilcon A showed full protection whilst eyes with Lotrafilcon A lens showed no protection. ${ }^{78}$ The full protection from Senofilcon A against UVB light is due to its polymer matrix being more oxygen permeable than Lotrafilcon A Other UV-blocking contact lenses were also evaluated in terms of their potential applications and efficacy. One of the clinically evaluated contact lenses in the market was Acuvue Contact lenses. ${ }^{79}$ Acuvue contact lens (Vistakon, Johnson \& Johnson Vision Products Inc., Florida) has been incorporated with a UV blocker within its polymer. This clinical evaluation was done in two phases where the first one involved a double-masked clinical trial to patients $(\mathrm{n}=94)$ followed by a randomised parallel group design. Patients $(n=61)$ wore Acuvue contact lens with UV blocker and the remaining patients $(n=33)$ wore conventional Acuvue contact lenses without UV blocker. Data analysis consisted of bio microscopic evaluations including vision assessment, comfort, handling and visual acuity. The aim of this two-phased clinical evaluation was achieved rendering no significant clinical differences with the addition of a UV blocking agent into the polymer matrix or affecting daily wear. ${ }^{79}$

Since then, studies have continuously been carried out to determine other potential applications of contact lenses, particularly in the field of medicine and therapeutics. One of the interesting findings was from a study conducted by Ciolino et al. ${ }^{80}$ The aim of the study was to design a contact lens with an antifungal medication incorporated to treat a fungal infection of the eye, Candida albicans. The lens was engineered to encapsulate the econazole-impregnated poly (lacticco-glycolic) acid (PLGA) on poly (hydroxyethyl methacrylate) (pHEMA) and then polymerised under UV light. Release studies were carried out and the experiment yielded extended antifungal activity against. ${ }^{80}$ There are various other applications of contact lenses that can be ventured on. In a review by Epstein 2012, therapeutic drug delivery via contact lens poses high potential in the management of eye conditions. The ability of contact lens to maintain antibiotic drug levels in the body makes it a great candidate to treat infections such as microbial keratitis. Treatment of glaucoma and allergy requires a sustained drug release formulation, reduced systemic absorption but with an increased localised bioavailability. Hence, this drug delivery system could facilitate the necessary requirements to manage these conditions. ${ }^{81}$ The aim of contact lens development was to initially correct vision. However, it is feasible that with technological advances, contact lenses could be used as a platform to capture images. These lenses could be incorporated with miniaturised cameras that are nontoxic and stable. Another advancement of contact lens could be the integration of photo detectors from graphene. This would enable wearers to see UV and infrared light. ${ }^{82}$ With the advancement of contact lens as one method of measuring IOP in the eyes, it could also potentially measure and monitor levels of other bodily biomarkers such as cytokines, chemical mediators, hormones, $\mathrm{pH}$ or body temperature. These potential breakthroughs could aid in the diagnosis and management of conditions that are not only associated with the eyes. ${ }^{83}$

\section{Current regulations on contact lenses}

Current regulations are executed by various regulatory agencies worldwide, Food and Drug Administration (FDA) in the United States, Medicines and Healthcare products Regulatory Agency (MHRA) in 
United Kingdom, Health Canada in Canada, and State Food and Drug Administration (SFDA) in China. In the UK, professional bodies namely general Optical Council, College of Optometrists, Association of British Dispensing Opticians, Association of Optometrists, and Association of Contact Lens Manufacturers work with the MHRA to regulate contact lenses. The Opticians Act 1989 Order 2005 covers the legislation for sales and fitting of both corrective and non-corrective contact lenses by a registered medical practitioner. Following a regulated optical assessment, the medical practitioner must issue a written contact lens specification for corrective contact lenses in accordance with the regulation. However, it is not a legal requirement for a written specification for non-corrective contact lenses, but it is a good practice if done accordingly. ${ }^{84,85}$ Currently, corrective contact lenses are regulated by the Medical Devices Directives of the European Commission, whilst non-corrective contact lenses are regulated under general product safety legislation. With the separate legislations for contact lenses, the MHRA initiated a consultation regarding the proposed regulations on medical devices by the European Commission in 2012. The proposed new regulation aims to extend the scope of the legislation on various medical devices and to introduce regulatory requirements on the reprocessing of single-use devices. These proposals would revise the European Union legislations, thus careful and strict considerations are necessary. This was a strategic step to attempt to resolve problems on the confusion and inconsistencies in regulating medical devices such as contact lenses. ${ }^{86,87}$ In the United States, the Federal Food, Drug and Cosmetic Act has been revised with the inclusion of Section 520 to provide information and guidelines on all types of contact lenses. This Act also established the need of a valid prescription for all types of contact lenses before it could be supplied to a consumer. Non-corrective lenses carry the same risks as those with corrective lenses, thus a safe regulation for both types was implemented. FDA has reviewed a number of non-corrective lenses and approved as cosmetics. These lenses were also approved for commercial distribution. However, not all were approved by the FDA; hence this was considered unlawful. As a result, FDA took action to pose strict guidelines on the regulation of contact lenses as devices. General control measures were implemented before the sale of non-corrective lenses including Quality System regulation approved premarket approval, exemption for investigational use (IDE) and other applicable regulations. ${ }^{51}$

\section{Hazards associated with medicated contact lenses}

It is an important aspect of drug and device manufacturing to ensure the safety and effectiveness of the materials when administered or used. The advancement of drug-eluting contact lenses aids in the monitoring of medical conditions. This development provides benefits to patients with better therapeutic outcome, compliance and adherence However, there are drawbacks in using contact lens as ocular drug delivery system. These drawbacks could potentially limit in vivo studies. Particularly with medicated contact lenses, in vivo studies have to be critically evaluated. Potential reactions of the contact lens matrix and the tear film could account to adverse effects. These could potentially lead to more harm. The major drawbacks affecting contact lens compliance are those from the lens itself. One of them is the lens fitting. Although comfortable initially, tight fitting can eventually cause uneasiness giving rise to tight lens syndrome. Similarly, loose fitting can cause the positioning to change with each blink causing fluctuations in vision. ${ }^{88}$ Another issue is poor lens care. This can be due to poor hygiene habits before and after use that may result in protein and lipid deposition. Microorganisms or even inert foreign bodies can contribute to these deposits and cause damage to the lens, which further causes irritation of the corneal eyes ${ }^{89}$ Furthermore, the importance of hygiene needs proper counselling especially to first time users, followed by proper training in lens care. This is the reason why eye specialists have to be hands-on in the fitting of the most suitable lens to users. In this way, they can monitor the either rationale for contact lens use or disease progression..$^{50,90}$ Improper or inappropriate wearing of contact lenses poses high risk of serious eye conditions. These include infections, corneal abrasions and corneal ulcers, which perceive high risk of blindness if left untreated. These periodic evaluations also allow justifications if the lenses need reconditioning or replacements. To evaluate the complications associated with contact lenses, Teo et al. ${ }^{91}$ collected data from public hospitals around Singapore. In 2year-period, around 953 patients were admitted to hospitals with contact lens related complications. Around $25 \%$ of the patients had infective keratitis, $24 \%$ suffered from epithelial keratitis, $18.8 \%$ were admitted with allergic conjunctivitis and less than $20 \%$ had dry eyes and neovascularization of the cornea. ${ }^{91}$

\section{Corneal abrasion}

Corneal abrasion is one of the most common risks of wearing contact lens. It is defined as superficial lesions in the anterior part of the eye, the cornea. The aetiology of corneal abrasion is mostly from contact lens wearers. This is because contact lens is worn very tightly in the eye. Potentially, removing the lens could pull off the epithelium. A retrospective study was conducted to investigate the incidence of corneal abrasion during contact lens wear involving keratoconic (KC) and non-keratoconic $(\mathrm{nKC})$ subjects. The subjects $(\mathrm{n}=494)$ were asked to visit the optometrists for regular check-ups. Out of all the subjects, 11 patients were diagnosed with corneal abrasion. Within the twomonths of the test period, 5 out of $68 \mathrm{KC}$ subjects and 6 out of $426 \mathrm{nKC}$ subjects were diagnosed with corneal abrasion. This study also evaluated the correlation of corneal abrasion incidence with the type of contact lens the subjects wore. Among the nKC patients, there was higher incidence of corneal abrasion in the subjects who used gas permeable lenses over hydrogel lenses ${ }^{92}$ The management of corneal abrasion has emphasised the discontinuation of contact lens use. ${ }^{93}$ A randomised controlled trial was conducted in patients $(n=88)$ aiming to investigate the effectiveness of non-steroidal antiinflammatory drugs (NSAIDs) for the treatment of corneal abrasions. The patients involved in this trial were treated with a single dose of cyclopentolate $0.5 \%$ and chloramphenicol eye ointment four times a day until the following day. These patients were grouped into two where one received topical ketorolac trometamol $0.5 \%$ ophthalmic solution and the other received placebo and served as the control group. The results of this randomised controlled trial revealed no significant difference between the treatment group and the control group 24hours post treatment. This showed that there was no relief of subjective symptoms such as pain, photophobia, grittiness, blurring of vision and watering of the eyes. One of the limitations of this trial was that the patients involved were diagnosed with corneal abrasion, but with no relation to contact lens wear. ${ }^{94}$ However, this could be used to support future studies that are associated to contact lens wear. Another option for the treatment of corneal abrasion is pressure patching. A comparative study was conducted to evaluate the treatment of corneal abrasion with and without pressure patching. ${ }^{95}$ It involved 201 patients with non-infected and non-contact lens related corneal abrasion. The subjects were grouped according to the cause of corneal abrasion on diagnosis; traumatic or secondary. The subjects were then treated with antibiotic ointment and mydriatics. The results showed that 
healing time for all subjects was faster, less painful, and fewer reports of blurred vision without the use of patches. There was however a similarity between the use and non-use of pressure patches, incidence of tearing and photophobia. ${ }^{95}$ Another association of contact lenses with corneal abrasion is its potential as a treatment. A clinical study was conducted in patients $(n=13)$ reported to have been diagnosed with corneal abrasion. Subjects were treated with a combination treatment of soft contact lens and Diclofenac (Voltaren) eye drops. The follow-up assessments to the subjects showed that there was a significant pain relief felt and the abrasions were healed within 3 days post insertion of contact lens. This treatment regime has the potential for treating corneal abrasion without the need for pressure patching. ${ }^{96}$

\section{Allergy and sensitivity}

Allergic reaction come into clinical presentation after second exposure to the allergen, which in context can either be the materials used or compositions of contact lens. If not the lens, allergy may occur due to the preservatives in contact lens (CL) care solutions or cleaning solutions. If not given proper attention, this can give rise to variants of conjunctival problems. Presentation of an allergic reaction includes excessive water discharge and redness of the eye. This could due to the increased permeation of vessels in the conjunctiva resulting to accumulation of pro-inflammatory mediators. Allergy and sensitivity can also be caused by allergens that are introduced right onto the surface of the eye. Examples of potential triggers are medications released from eye formulations and contact lens solutions. ${ }^{97}$ This allergic reaction is typically cell-mediated (Gell-Coombs type IV) reactions. This autoimmune response induced by cell proliferation and metabolism of cytokines is followed by apoptosis of target cells. These result in unfavourable side effects to the host i.e. the eye in attempts to protect it. ${ }^{98}$ In simpler terms, under normal conditions, only trace levels of cytokines and other inflammatory mediators are found in the tear film. However, when there is an environmental stress such as contact lens wear, there could be an increase in the expression of these mediators which then results to tear film dysfunction..$^{99,100}$ Ocular allergy is usually considered by ophthalmic practitioners as Type I hypersensitivity. The mechanism of ocular allergy is mediated by immunoglobulin IgE. A theory is discussed in 92 studies that proposed four cascade reactions provoking ocular allergy namely; sensitisation, mast cell degranulation, activation and late phase response. In an ocular allergic incident, the mast cells which are the primary cells involved travel through to the tissue layers. In here, these mast cells get degranulated and then release histamine into blood stream and causes itching. ${ }^{101}$ It is believed that $15-20 \%$ of the world population has encountered allergic reaction and around 40$60 \%$ of these allergy- conditions are contact lens related. This high percentage could be caused by the contact lens itself or the contact lens solutions used for cleaning the lenses. ${ }^{100}$ A proposed treatment for ocular allergy caused by contact lenses was the use of a topical anti-allergic medication branded as Patanol, clinically known as olopatadine hydrochloride $0.1 \%{ }^{102}$ This medication was clinically evaluated and tested in patients with allergic conjunctivitis caused by contact lenses (group I) and seasonal allergic conjunctivitis (group 2). The dosage regime for Patanol indicated instilling a drop into each eye twice daily which is then monitored weekly until the $4^{\text {th }}$ week. It was found out that the symptoms of the ocular allergy such as itching and tearing reached the mild stage post-treatment for group I. It was reported to have reduced the symptoms by more than $50 \%$ in this study. It was concluded that Patanol could potentially be the treatment of choice for contact lens-induced allergy. ${ }^{102}$

\section{Contact lens-associated papillary conjunctivitis (CLAPC)}

CLAPC is clinically defined as the presence of calcium deposits in the corneal surface. CLAPC is a type of allergic reaction caused by a particular allergen that is the organic material found on the surface of contact lens. CLAPC is accompanied by blurred vision, excessive mucus secretion, and itching. It is known that the incidence of CLAPC increases proportionally with increase in duration of wearing contact lens. Statistics estimated that $1-5 \%$ of soft contact lens wearers and $1 \%$ of hard lens wearers will develop CLAPC. There are four goals of managing CLAPC: removal of deposits in the lens, reduce exposure time of wearing, proper wearing regimen including suitable materials, and medical therapy. ${ }^{97}$ The aim of contact lens was to initially manage blurred vision. Consequently, contact lens can also be of risk factor for blurred vision. Contact lens was designed to provide great acuity for the wearer especially at first use. However, some factors are associated to cause blurry vision. Deposits on the surface of contact lens may result to hazy vision. Although the eyelids can act as windshield wipers, it is still very important to a follow proper cleaning regimen before using contact lens to avoid infections. Another factor could be when the contact lenses are not properly stilled on the centre of the eye. If the lens keeps on moving, there is most likely going to be blurriness and potentially may cause corneal abrasion. ${ }^{103}$ It is believed that silicone based contact lenses are associated with developing CLPC and this is attributed to the interaction between the silicone hydrogel and the preservative content in the multipurpose care regimens used. ${ }^{104}$ Substituting the latter with preservative-free Clear Care can improve CLPC Smythe. ${ }^{105}$ Sorbara et al. ${ }^{106}$ study demonstrated that patients using silicone hydrogel contact lenses for treating hyperaemia and neovascularization developed CLPC possibly because of surface properties of the contact lenses, modulus values, frequency of replacement or lens care. Hence frequent replacement and regular check and fellow up visit will help reduce the incidence of papillary conjunctivitis. ${ }^{106}$ In severe cases, combination of different factors associated with contact lens wearing or the engineering of contact lens may pose high risk of infection.

\section{Contact lens-associated microbial keratitis (CLMK)}

CLMK is another common corneal complication reportedly caused by overnight lens wearing or inappropriate cleaning. Both the lens type and wearing schedule affect the incidence rates of CLMK in contact lens wearers. It is believed that in every 10,000 contact lens wearers 25.4 of extended wear of silicone hydrogel lenses get CLMK. ${ }^{107}$ The most common pathogens for this type of infections are Pseudomonas aeruginosa and Staphylococcus aureus. These types of bacteria were also the findings of a study conducted in 2008 where corneal scrapings collected from patients presenting with CLMK $(n=239)$ were analysed. ${ }^{108}$ This contact lens-associated complication is commonly presented with pain in the eyes, eye redness, blurred vision, excessive tearing, and presence of eye discharge. In the US, approximately 25,000 Americans develop this type of infection yearly. This infection can be managed and treated with a course of antibiotics such as second- generation fluoroquinolones, Moxifloxacin and Gatifloxacin. ${ }^{108-110}$ The retrospective study conducted by Johan et al., ${ }^{11}$ at Rotterdam Eye Hospital evaluated the epidemiological characteristics, clinical presentation, isolated organism, and treatment for all hospitalised patients with CLMK. The study also revealed that more than $65 \%$ of the isolated microorganisms were Pseudomonas aeruginosa with $2.7 \%$ and $1.3 \%$ resistance to gentamicin and ofloxacin respectively. ${ }^{111}$ Almost $65 \%$ of the admitted cases showed 
best corrected visual acuity (BCVA) lower than 0.05 Snellen. Other pathogens such as acanthamoeba and fungi were also reported to be associated with CLMK.${ }^{95}$ With the incorporation of drugs into contact lenses, the safety, stability and efficacy must be strictly studied. It has to be ensured that the drugs are compatible with the lens composition to avoid any further eye conditions and to prevent any risk of blindness.

\section{In Vivo studies on medicated contact lenses}

Majority of the ocular administrations are eye drops. However, with the advancement of research, contact lenses are slowly evolving and taking over conventional methods. Over theyears, the scope of ocular drug delivery has extended to contact lenses..$^{59}$ Initially, this started with incorporation of drugs through the soaking method. The method involves submerging the lenses into a drug solution for a fixed period of time. This allows adsorption of drug molecules onto the polymer matrix of the contact lens. A study using Beagle dogs portraying the administration of Timolol through this non-invasive method for the treatment of glaucoma is one of the few that compares its efficacy. Glaucoma affects about 60.5 million worldwide. The management of the disease using contact lenses offers less hassle and also limits room for error. This is made possible by terminating the requirement for intricate details like positioning of the drops on the eye, the correct time intervals between doses and the number of administrations. Along with the therapeutic effectiveness and reduced side effects, the use of contact lens helps increase the patient compliance majorly. The study also shed light on the comparative efficacy of contact lenses against eye drops. However, more reduction in IOP is not true for all contact lenses. In fact, there was no significant difference in efficacy between eye drops administered at regular intervals and a drug loaded Pure ACUVUE® Tru EyeTM. ${ }^{112}$ Only when the contact lenses were manufactured with a sustained release formulation (ACUVUE® Tru EyeTM loaded with $0.23 \mathrm{~g} / \mathrm{g}$ vitamin), was it able to significantly reduce the IOP. This was achieved by increasing the duration of time to release the drugs, fromhours todays. Furthermore, the study revealed that vitamin $\mathrm{E}$ is not released into the tear fluid. From further observation of the lens packaged in PBs solution, it was confirmed that the vitamin E composition in the lens stays intact under the influence of tear proteins. ${ }^{12}$ A more recent study that aimed to incorporate drugs in the polymer matrix involved testing of contact lenses with drug polymer films. A solution containing Latanoprost and Poly (lacticco- glycolic) acid (PLGA) was latched onto the dry lens using a spin coater. These lenses were designed to achieve a sustained release formulation. These were tested in New Zealand white rabbit eyes and observed for over a month. However, the reduction of IOP could not be quantified as the drug molecules do not work in the same fashion as in humans. This was due to the variation in dimensions of rabbit eyes to humans. Another reason was that the contact lenses were designed for humans; hence this should have been considered before the study. In this study, drug molecules were absorbed into the aqueous humour. The level of drug molecules was sustained for a minimum of 24days without any redness, discomfort or even toxicity. There was however an initial burst of drug molecules from the lenses and this was considered a limiting factor. Furthermore, the results from in vitro experiments showed correlation with the in vivo studies. Replacement of contact lenses every month could mean a major advancement in therapeutic efficacy along with patient compliance. This removes the need of assistance and ensures correct administration. ${ }^{63}$ The potential for contact lenses in treating diseases has lead formulation scientists to further study its use in improving minor conditions. One example could be the incorporation of dinucleotides in the management of dry eyes. Among the comparison of various different contact lenses, nonionic lenses (Comfilcon A) had the longest (RT50) retention time as reported in an in vivo study on New Zealand white rabbit eyes. ${ }^{63}$ It was an interesting feature that ionic charge on the lenses was superior to water content and hydrophilicity. Negative charge on the lenses was a result of the negative charges presented by Ap4A at neutral $\mathrm{pH}$. Clinically, this would cause side effects due to the same charges in the body. This superiority influenced the release of dinucleotide and hence, the drug molecules. ${ }^{113}$

\section{Regulations and implications of medicated contact lenses}

The composition of contact lenses used for both correcting vision and ocular delivery include monomers such as HEMA (poly (hydroxyethyl methacrylate)) and MA (methacrylic acid). However, poly (lactic-co-glycolic) acid (PLGA) is also one of the approved ones by the FDA. PLGA is commonly used for encapsulation of the drug in the polymer films. The availability of polymers in the manufacture of contact lenses is not abundant at present times because these polymers fall under a Class (II) FDA Regulations indicating a moderate health risk. ${ }^{114} \mathrm{~A} 10$ year pivotal study evaluated the evidence that was used to approve the ophthalmic drugs and high-risk medical devices over the past decade. After contact lenses were classed as medical devices in 2012, regulations have evolved massively with strict considerations to health risks and benefits. FDA approved 11 drugs for ophthalmic delivery and 25 medical devices. Clinical trials for ophthalmic therapeutics included randomisation, masking and comparison to placebo. These trials were cross-analysed to determine its significance in the approval of marketing regulation for both the drug and medical devices. The results showed that there were a significantly higher percentage of randomised and masked clinical trials carried out for testing ophthalmic drugs than the medical devices. However, drugs as well as devices were less likely to be approved for well-established conditions even with larger randomised controlled trials. Another aspect this study looked at was the constant change in regulators and their decision-making. Regulators were shown to be more flexible in making decisions provided that there is a good risk benefit balance. This has provided the manufacturers with the opportunity to develop more treatment options for consumers. Similarly, safety and efficacy was another aspect that was studied. Upon sanction, post-approval studies were carried out to determine risks and adverse effects. Out of the 8 post approval studies for medical devices, six focused on the safety and the other two on effectiveness. Furthermore, not only were six of post-approval studies for ophthalmic drugs but it was also a larger cohort with inclusion of paediatric patients as well. ${ }^{115}$

\section{Conclusion}

To conclude, despite the setbacks of contact lenses in certain aspects like hypersensitivity and physical incompatibility, they still undoubtedly pose a great potential as ocular drug delivery system. With the development of new contact lenses for a variation of consumers, the number of wearers has gradually increased overtime. With the continuous advancement of research in this field, there is a high hope for contact lenses to be used as an ocular drug delivery system for the masses. In the long term, this means that contact lens could potentially provide a great platform for managing conditions such as glaucoma, allergy, and other photosensitive conditions. Moreover, the 
use of smart contact lenses in managing non- ocular conditions like diabetes demonstrates its endless possibilities. However, different regulatory bodies are very vigilant with these new approaches. This is undoubtedly considered as a big hurdle for new innovations to be put on the market and used by consumers. Nevertheless, regulatory bodies recognise the contribution contact lenses can make to improve the health of the public. Hence, in the long term with more evidence contact lenses as ocular drug delivery system will be considered an effective means for diagnosis and management of conditions.

\section{Acknowledgements}

Authors would like to acknowledge the funding from Kingston University's Access Working Group for funding and supporting the project.

\section{Conflict of interest}

Author declares that there is no conflict of interest.

\section{References}

1. Young T. The Development of Contact Lenses. Health Case Study 31: The Contact Lens Industry: Structure, Competition, and Public Policy. 1979;31:9-12.

2. Siviglia N. History of Contact Lenses. Edward Hand Medical Heritage Foundation. 1964;47:45-48.

3. Bergin J. Contact Lens Polymers. Introduction to Polymers CE435. 2000:1-15.

4. Boyd B. Modern Ophthalmology: The Highlights. JayPee-Highlights Medical Ltd; Republic of Panama (p460). 2011.

5. Schifrin L, Rich W. Health Technology Case Study 31, OTA-HCS-31. The Contact Lens Industry: Structure, Competition, and Public Policy. Washington: DIANE Publishing; 1984:1-63.

6. Nelson R, Ballhaus W, Farrisey R. Federal policies and the medical devices industry. Washington: DIANE Publishing; 1984:107.

7. Weissman B. Clinical Contact Lens Practice. American Optometrists Association. Philadelphia: Lippincott Williams \& Wilkins; 2005:1072.

8. Nicolson PC, Vogt J. Soft contact lens polymers: An evolution. Biomaterials. 2001;22(24):3273-3283.

9. Bengani LC, Hsu K-H, Gause S, et al. Contact lenses as a platform for ocular drug delivery. Expert Opin Drug Deliv. 2013;10(11):1483-1496.

10. The Difference between a Contact Lens and Spectacle Prescription. 2012.

11. Contact Lens Manufacturers Association (CLMA). Wouldn't you rather wear contact lenses than glasses? GP Contact Lenses. 2013.

12. CooperVision. How contact lenses work. CooperVision UK. 2015.

13. Benefits of Vision Correction with Contact Lenses. Centers for Disease Control and Prevention. 2015.

14. Contact lenses and myopia control in children. British Contact Lens Association. 2015.

15. Bailey G, Heiting G. Myopia (Nearsightedness) Definition, Causes and Treatment. All About Vision. 2014.

16. Facts about Myopia. National Eye Institute. 2015.

17. Walline JJ, Greiner KL, McVey ME, et al. Multifocal contact lens myopia control. Optom Vis Sci. 2013;90(11):1207-1214.
18. Kading D, Mayberry A. Slowing myopia progression in children. Review of Optometry. 2012

19. Katz J, Schein OD, Levy B, et al. A randomized trial of rigid gas permeable contact lenses to reduce progression of children's myopia. Am J Ophthalmol. 2003;136(1):82-90.

20. Walline JJ, Jones LA, Chitkara M, et al. The Adolescent and Child Health Initiative to Encourage Vision Empowerment (ACHIEVE) study design and baseline data. Optom Vis Sci. 2006;83(1):37-45.

21. Guthrie S. Effect of dual-focus soft contact lens wear on axial myopia progression in children. Contact Lens Update. 2011.

22. Facts about Hyperopia. National Eye Institute. 2015.

23. Long sight-hypermetropia: Causes of long-sightedness. Patient.co.uk. 2015.

24. Universitat Politècnica de Catalunya Contact lens capable of correcting hyperopia without surgery. Science Daily. 2010.

25. Emara KE, Abdulsalam O Al, Habash A Al. Secondary implantation of implantable collamer lens (ICL) for correction of anisometropic hyperopia in a 3-year-old pseudophakic child. Saudi J Ophthalmol. 2016;30(1):75-77.

26. Siganos DS, Siganos CS, Pallikaris IG. Clear lens extraction and intraocular lens implantation in normally sighted hyperopic eyes. $J$ Refract Corneal Surg. 1994;10(2):114-117.

27. Facts about Astigmatism. National Eye Institute. 2015.

28. Astigmatism and contact lenses. British Contact Lens Association. 2015.

29. Contact Lens Manufacturer Association. Correcting Astigmatism with GP Contact Lenses. GP Contact Lenses. 2013.

30. Cole J, Mullarkey C. Torics for Astigmatism: Soft Lenses, Hard Choices. Review of Optometry. 2015.

31. Young G, Sulley A, Hunt C. Prevalence of astigmatism in relation to soft contact lens fitting. Eye Contact Lens. 2011;37(1):20-25.

32. Weissman B, Barr J, Harris M, et al. Optometric Clinical Practice Guideline Care of the Contact Lens Patient, 2e. American Optometric Association. 2006

33. Facts and Stats on the UK contact lens market. British Contact Lens Association. 2015.

34. Keay L, Edwards K, Naduvilath T, et al. Microbial keratitis predisposing factors and morbidity. Opthalmology. 2006;113(1):109-116.

35. Nilsson SE, Montan PG. The hospitalized cases of contact lens induced keratitis in Sweden and their relation to lens type and wear schedule: results of a three-year retrospective study. CLAO J. 1994;20(2):97-101.

36. Hart DE, Bennett E, Weissman B. Deposits and coatings hydrogel lens/ tear film interactions. Clinical Contact Lens Practice. Philadelphia: JV Lippincott Company; 1991.

37. Rosenthal RA, Henry CL, Schlech BA. Contribution of regimen steps to disinfection of hydrophilic contact lenses. Cont Lens Anterior Eye. 2004;27(3):149-156.

38. List of Contact Lenses. Center for Devices and Radiological Health. 2014 .

39. Appendix A. FDA Approved Contact Lenses and Current prices. US Food and Drugs Administration FDA. 1983.

40. ACUVUE® ADVANCE®. Johnson and Johnson Vision Care Companies. 2015. 
41. Astigmatic Contact Lens- nSoft Lens Toric Contact Lenses. Bausch+Lomb, USA; 2015.

42. DAILIES® Contact Lenes by Alcon. Dailies. 2015.

43. Proclear Contact Lenses. CooperVision. 2015.

44. SaturEyes Multifocal. Metro-Optics. 2015.

45. Purevision Contact Lenses. Bausc and Lomb, USA; 2015.

46. Get the best price on Softmate B Contact Lenses. Lensshopper. 2015.

47. Tresoft. The Right Contact. 2015.

48. Hydrogenics. Hydorgenics 60 by CooperVision. 2015.

49. Types of contact lenses. Rochester Eye Associates. 2014.

50. FDA Contact Lenses- Decorative Contact Lenses. Center for Devices and Radiological Health. 2015.

51. Smith E. Guidance for Industry. FDA staff, Eye Care Professionals, and Consumers: Decorative, Non-corrective contact lenses. 2006.

52. Steinemann TL, Fletcher M, Bonny AE, et al. Over-the-counter decorative contact lenses: Cosmetic or Medical Devices? A Case Series. Eye Contact Lens. 2005;31(5):194-200.

53. Singh S, Satani D, Patel A, et al. Colored cosmetic contact lenses: an unsafe trend in the younger generation. Cornea. 2012;31(7):777-779.

54. Sauer A, Bourcier T. Microbial keratitis as a foreseeable complication of cosmetic contact lenses: a prospective study. Acta Ophthalmol. 2011;89(5):e439-42.

55. Cosmetic Contact Lenses. British Contact Lens Association. 2015.

56. Kumar A, Jha G. Drug delivery through soft contact lenses: An introduction. Chronicles of Young Scientists. 2011;2(1):3.

57. Credille BS, Daniel F, Breeden J. Smart contact lenses could make eye drops a thing of the past. Medical Xpress; 2011:1-2.

58. Hui A. Contact lenses for drug delivery- overview and recent developments. Contact Lens Update. 2012.

59. Elshaer A, Ghatora B, Mustafa S, et al. Contact lenses as drug reservoirs \& delivery systems: the successes and challenges. Ther Deliv. 2014;5(10):1085-1100.

60. Campbell-Burns D, Lebow K. Contact Lens Update 1983-1996. Contact Lens Anterior Eye. 1998;21(3):95-96.

61. Kim J, Conway A, Chauhan A. Extended delivery of ophthalmic drugs by silicone hydrogel contact lenses. Biomaterials. 2008;29(14):2259-2269.

62. Guidi G, Hughes TC, Whinton M, et al. The effect of silicone hydrogel contact lens composition on dexamethasone release. J Biomater Appl. 2014;29(2):89-94.

63. Ciolino JB, Stefanescu CF, Rose AE, et al. In vivo performance of a drug-eluting contact lens tp treat glaucoma for a month. Biomaterials. 2014;35(1):432-439.

64. Jain MR. Drug delivery through soft contact lenses. Br J Ophthalmol. 1988;72(2):150-154.

65. Xinming L, Yingde C, Lloyd AW, et al. Polymeric hydrogels for novel contact lens-based ophthalmic drug delivery systems:A review. Cont Lens Anterior Eye. 2008;31(2):57-64.

66. Gulsen D, Chauhan A. Ophthalmic drug delivery through contact lenses. Invest Ophthalmol Vis Sci. 2004;45(7):2342-2347.

67. Twa MD, Roberts CJ, Karol HJ, et al. Evaluation of contact lens-embedded sensor dor intraocular pressure measurement. J Glaucoma. 2010;19(6):382-390.
68. Anton A, Neuburger M, Böhringer D, et al. Comparative measurement of intraocular pressure by Icare tonometry and Airpuff tonometry in healthy subjects and patienst wearing therapeutic soft contact lenses. Graefes Arch Clin Exp Ophthalmol. 2013;251(7):1791-1795.

69. Barras C. Smart contact lens feels the pressure of glaucoma. New Scientist. 2008.

70. Cong H, Pan T. Photopatternable conductive PDMS materials for microfabrication. Advanced Functional Materials. 2008;18(13):1912-1921.

71. Legerton J. The Future is Now: Unveiling Smart Lenses. Review of Contact Lenses. 2011.

72. SENSIMED Triggerfish $®$. Sensimed, Switzerland; 2014.

73. Mansouri K, Shaarawy T. Continuous intraocular pressure monitoring with a wireless ocular telemetry sensor: initial clinical experience in patients with open angle glaucoma. Br J Ophthalmol. 2011;95(5):627-629.

74. BBC News. Google unveils "smart contact lens" to measure glucose levels. BBC NEWS. 2014.

75. World Health Organization. Health effects of UV radiation. WHO. 2015.

76. UV Protection with contact lenses. American Optometric Association. 2014.

77. Summary of Safety and Effectiveness data. Food and Drug Administration. 2005.

78. Giblin FJ, Lin L-R, Leverenz VR, et al. A class I (Senofilcon A) Soft contacxt lens prevents UVB-induced ocular effects, including cataract, in the rabbit in vivo. Invest Ophthalmol Vis Sci. 2011;52(6):3667-3675.

79. Hickson-Curran S, NAson R, Becherer D, et al. Clinical evaluation of ACUVUE contact lenses with UV blocking characteristics. Optom Vis Sci. 1997;74(8):632-638.

80. Ciolino JB, Hudson SP, Mobbs AN, et al. A prototype antifungal contact lens. Invest Ophthalmol Vis Sci. 2011;52(9):6286-6291.

81. Epstein A. Technological advances in drug delivery. Review of Cornea and Contact Lenses. 2012

82. Liu C-H, Chang Y-C, Norris TB, et al. Graphene photodetectors with ultra-broadband and high responsivity at room temperature. Nature $\mathrm{Na}$ notechnology. 2014;9(4):273-278.

83. Hale M. Towards 2020: Contact lenses of the future. Optician: contact lenses, feature, news and features. 2014.

84. Articles AOF 2005 No 848. Health Care and Associated Professions Opticians. The Opticians Act 1989 (Amendment) Order 2005. 2005:1989(848)

85. Regulations on contact lens fitting and supply. British Contact Lens Association. 2015.

86. Response to MHRA Consultation on the Revision of European Legislation on Medical Devices. Medicines and Healthcare Products regulatory Agency. 2013

87. The revision of European Legislation on Medical Devices. Medicines and Healthcare products Regulatory Agency MHRA. 2012.

88. Olivia S, Tidy C. Contact Lens Problems. Patient info. 2015.

89. Schroeder H. Contact Lens Problems. Eye Ear Nose Throat Monthly. $1950 ; 29(8): 422-423$.

90. Center for Devices and Radiological Health. Contact Lens Risks. Food and Drugs Administration. 2014

91. Teo L, Lim L, Tan DTH, et al. A survey of contact lens complications in Singapore. Eye Contact Lens. 2011;37(1):16-19. 
92. Chun M, Weissman B, Barnhart L. Corneal abrasion associated with contact correction of keratoconus- a retrospective study. American Academy of Optometry. 1994.

93. Shahid SM, Harrison N. Corneal abrasion: assessment and management. InnovAiT: Education and Inspiration for General Practice. 2013;6(9):551-554.

94. Goyal R, Shankar J, Fone DL, et al. Randomised controlled trial of ketorolac in the management of korneal abrasions. Acta Ophthalmol Scand. 2001;79(2):177-1779.

95. Kaiser PK. A comparison of pressure patching versus no patching for corneal abrasions due to trauma or foreign body removal: Corneal abrasion patching study group. Ophthalmology. 1995;102(12):1936-1942.

96. Salz JJ, Reader AL, Schwartz LJ, et al. Treatment of corneal abrasion swith soft contact lenses and topical diclofenac. J Refract Corneal Surg. 1994;10(6):640-646.

97. Tuft S. Allergy and sensitivity as a result of contact lens wear. Continuing Education. 1999:28-34.

98. Rajan TV. The Gell-Cooms classification of hypersensitivity reactions: a re-interpretation. Trends Immunol. 2003;24(7):376-379.

99. Gayton JL. Etiology, prevalence and treatment of dry eye disease. Clin Ophthalmol. 2009;3:405-412.

100. Urgacz A, Mrukwa E, Gawlik R. Adverse events in allergy sufferers wearing contact lenses. Postepy Dermatol Alergol. 2015;32(3):204-209.

101. Schachet J. Treating allergy in contact lens patients. Contact Lens Spectrum. 2007.

102. Brodsky M. Allergic conjunctivitis and contact lenses: experiences with Olopatadine hydrochloride $0.1 \%$ therapy. Acta Ophthalmol Scand Suppl. 2000;(230):56-59.

103. Why is my vision blurry with contact lenses. 1-800 Contacts. 2015.

104. Jones L, MacDougall N, Sorbara LG. Asymptomatic corneal staining associated with the use of Balafilcon silicone-hydrogel contact lenses disinfected with a polyaminopropyl biguanide-preserved care regimen. Optom Vis Sci. 2002;79(12):753-761.
105. Smythe JL. Caring for silicone hydrogels. Contact Lens Spectrum. 2003:18-23.

106. Sorbara L, Jones L, Williams-Lyn D. Contact lens induced papillary conjunctivitis with silicone hydrogel lenses. Cont Lens Anterior Eye. 2009;32:93-96.

107. Stapleton F, Keay L, Edwards K, et al. The incidence of contact lens-related microbial keratitis in Australia. Ophthalmology. 2008;115(10):1655-1662.

108. Ormerod LD, Smith RE. Contact lens-associated microbial keratitis. Archives of Ophthalmology. 1986;104(1):79-83.

109. Basics of bacterial Keratitis. Centers for Disease control and Prevention. 2015.

110. Murillo-Lopez FH. Bacterial keratitis: background, pathophysiology, epidemiology. Medscape. 2014.

111. Hoddenbach JG, Boekhoorn SS, Wubbels R, et al. Clinical presentation and morbidity of contact lens-associated microbial keratitis: a retrospective study. Graefes Arch Clin Exp Ophthalmol. 2014;252(2):299-306.

112. Peng CC, Burke MT, Carbia BE, et al. Extended drug delivery by contact lenses for glaucoma therapy. I Control Release. 2012;162(1):152-158.

113. Hui A, Willcox M, Jones L. In Vitro and In Vivo evaluation of novel Ciprofloxacin- releasing silicone hydrogel contact lenses. Invest Ophthalmol Vis Sci. 2014;55(8):4896.

114. Farandos NM, Yetisen AK, Monteiro MJ, et al. Contact lens sensors in ocular diagnostics. Adv Healthc Mater. 2015;4(6):792-810.

115. Hwang J, Hwang TJ, Ciolino JB. Pivotal clinical trials of novel ophthalmic drugs and medical devices: retrospective observational study, 2002-2012. British Medical Journal. 2015;5(6):e7987. 